\title{
ESTUDO COMPARATIVO ENTRE O MÉTODO CONVENCIONAL E O MÉTODO DA PEROXIDASE ANTI-PEROXIDASE NA PESQUISA DO PARASITISMO TISSULAR NA CARDIOPATIA CHAGASICA CRONICA (")
}

A. J. A. Barbosa (1), H. GOBbi (1), B. T. LINo (1), E. LAGES-Silva (2), L. E. RAMTREZ (3),

V. P. A. TELXEIRA (4) e H. O. ALMEIDA (4)

\begin{abstract}
R E S U M O
Na maioria dos chagásicos crônicos o Trypanosoma cruzi não é detectado no tecido ou apresenta se com extrema raridade, mesmo quando é pesquisado exaustivamente. Sendo os métodos utilizados, até então, inespecíficos para a demonstração do T. cruzi, propôs-se no presente trabalho proceder ao estudo comparativo entre o método convencional $(\mathrm{HE})$ e o método imunocitoquímico pela peroxidase anti-peroxidase (PAP), na avaliação quantitativa do parasitismo. Selecionaram-se 3 casos de cardiopatia chagásica crônica e, de um mesmo fragmento de cada caso, obtiveram se cortes que foram corados pelo H.E. (média de 100 cortes por caso) e, consecutivamente, outros que foram corados pelo PAP (média de 70 cortes por caso). O caso n. 1 foi autopsiado em 1952 e apresentava parasitismo frequiente. Nos demais, o exame rotineiro foi negativo. Obtiveram-se os seguintes resultados expressos em $n .{ }^{\circ}$ de ninhos $/ 100$ cortes, respectivamente, corados pelo H.E. e pelo P.A.P. (HE/PAP). Caso n. $1=80 / 171$; caso n. $2=5 / 116$ e caso $n .{ }^{\circ} 3$ $=1 / 2$. Os resultados mostram que o método imunocitoquímico empregado, além de facilitar o diagnóstico do parasitismo, demonstra também pequenos ninhos de amastigotas que dificilmente seriam diagnosticados pelos métodos convencionais; além disso mostrou-se útil mesmo em tecido incluídos em parafina há longo tempo.
\end{abstract}

UNITERMOS: Moléstia de Chagas - PAP - Cardiopatia chagásica crônica Parasitismo cardíaco.

\section{N T ROD U G A O}

Admite-se ser extremamente rara a presenca do Trypanosoma cruzi nos tecidos da quase totalidade dos portadores de doença de Chagas crônica ${ }^{7}$. No coraçāo, principal órgão de choque da doença, apesar de muitas vezes apresentar miocardite relativamente acentuada, não se de- monstra a presença do parasita, pelo menos através de exames histológicos rotineiros ${ }^{3}$. Por outro lado, quando se procedem a exames sistematizados tanto do coração ', como de outros órgăos 2,6 a frequiência dos achados do parasita aumenta, às vezes inesperadamente, demons.

(1) Departamento de Anatomia Patológica e Medicina Legal da Faculdade de Medicina da UFMG. Ay. Alfredo Batena 190. Belo Horizonte, CEP 30000, MG - Brasil

(2) Centro de Pesquisa René Rachou. FIOCRUZ, Belo Horizonte, MG - Brasil

(3) Departamento de Parasitologia; Universidade de Antioquia, Medellin, Colombia

(4) Disciplina de Patologia Geral da Faculdade de Medicina do Triângulo Mineiro, Uberaba, MG, Brasil

(*) Trabalho realizado com auxilio tinanceiro da FINEP e CNPq

Endereço para correspondéncia e pedidos de Separatas: Alfredo J. A. Barbosa, Faculdade de Medicina da UFMG Departamento de Anatomia Patológica e Medicina Legal, Av. Prof. Alfredo Balena, 190, $30000-$ Belo Horizonte - Brasil 
BARBOSA, A. J. A.; GOBBI, H.; LINO, B. T.; LAGES-SILVA, E.; RAMLREZ, L. E.; TEIXEIRA, V. P. A. \& ALMEIDA, H. O. - Estudo comparativo entre o método convencional e o método da peroxidase anti-peroxidase na pesquisa do parasitismo tissular na cardiopatia chagásica crônica.

Rev. Inst. Med. trop. Sảo Paulo, 28:91-96, 1986.

trando que, pelo menos em um certo número de casos, o parasitismo tissular pelo $\mathbf{T}$. cruzi pode ser mais frequiente do que o correntemente admitido. O principal ponto sujeito a criticas nos trabalhos que lidam com a pesquisa do parasita nos tecidos é o fato de todos eles utilizarem metodologias convencionais, geralmente a coloração pela Hematoxilina e Eosina (HE), inespecífica para a demonstração do $\mathbf{T}$. cruzi, pois, cora também outras estruturas celulares basofilicas como núcleos, fragmentos de núcleos, certos corpúsculos intracelulares e mesmo outros parasitas morfologicamente semelhantes ao T. cruzi como Toxoplasma e Histo. plasma capsulatum. Portanto, o diagnóstico do parasitismo tissular somente pode ser feito com segurança pelos métodos convencionais quando se encontram ninhos de amastigotas intracelulares relativamente grandes e com os parasitas exibindo suas caracteristicas anatômicas peculiares. Ninhos menores, ou pouco típicos, ou mesmo formas isoladas do parasita, geralmente passam despercebidas ou não oferecem elementos suficientes para diagnóstico.

Com a recente utilização de método imu nocitoquímico pela peroxidase anti-peroxidase (PAP) para a demonstração do $\mathbf{T}$. cruzi em tecidos incluidos em parafina ${ }^{5}$ abrem-se as por tas de uma metodologia nova e altamente específica para estudo do parasitismo tissular na doença de Chagas humana e na tripanosomose cruzi experimental. Entretanto, sendo o método relativamente mais caro e complexo que os rotineiros, a sua utilização para quantificar o farasitismo em tecidos de chagásicos crônicos somente valeria a pena se, além de facilitar o diagnóstico e ser mais específico, também de monstrasse maior número de parasitas, aumen tando sensivelmente a probabilidade do diagnóstico dos mesmos e revelando formas parasitárias que não poderiam ser evidenciadas pelos métodos convencionais. No sentido de verificar esta possibilidade realizou-se o presente trabalho.

\section{MATERIAL E METODO}

Selecionaram-se três cardiopatias chagásicas crônicas de pacientes falecidos com insuficiência cardíaca congestiva e que apresentavam exame de Machado-Guerreiro positivo. Duas foram de indivíduos autopsiados na Faculdade de Me- dicina do Triângulo Mineiro, Uberaba. M.G.: $\mathrm{N}-3404$, sexo masculino, 37 anos, mulato, peso do coração $350 \mathrm{~g}$ e N-2891, sexo masculino, 40 anos, branco, peso do coração $780 \mathrm{~g}$. O terceiro caso foi autopsiado na Faculdade de Medicina da UFMG, Belo Horizonte, M.G., em abril de 1952 sendo a autópsia de número A-804, referente a individuo de sexo feminino, 24 anos. de cor preta, peso do coração $350 \mathrm{~g}$. O quadro histológico da miocardite foi aquele classicamente descrito na doença de Chagas crônica, tendo sido semelhante nos três casos, exceto para o fato de que o caso A-804 apresentava parasitismo relativamente frequiente, motivo pelo qual foi escolhido. Nos outros dois casos evidenciou-se parasitas somente após exame de cortes seriados.

De cada caso escolheu-se 1 fragmento de tecido incluído em parafina, contendo toda a espessura da parede ventricular (A-804) ou um anel de átrio direito incluindo septo interatrial (N-2891 e $\mathrm{N}$-3404). Obteve-se o seguinte número de cortes semi seriados de cada bloco e que foram corados pelo HE: A-804, 110 cortes; N-2891, 100 cortes e N-3404, 100 cortes. Em seguida, obteve-se de cada caso o seguinte número de cortes semi seriados que foram utilizados para a coloração pelo método de peroxidase anti-peroxidase: A-804, 71 cortes; N-2891, 100 cortes e N.3404, 61 cortes. Para a aplicação do método da imunoperoxidase utilizou-se anti-soro primário produzido em coelho pela inoculação i.p. de $10^{7}$ tripomastigotas da cepa $\mathrm{Y}$ provenientes de camundongo irradiado. $O$ anti soro utilizado, de n. 41 . apresenta título de anticorpos anti- T. cruzi de $1: 1280$ à imunofluorescência indireta e alta taxa de anticorpos líticos anti- $\mathbf{T}$ cruzí, como evidenciada pela reação de lise mediada por complemento ${ }^{9}$. O anti-soro foi diluído em tampão fosfato salino (PBS), $\mathrm{pH} 7,2$ na proporção de 1:1000 e aplicado sobre os cortes que foram incubados em câmara úmida a $4^{\circ} \mathrm{C}$ por 18 horas, conforme utilizado em trabalho anterior ${ }^{5}$. Anteriormente a esta primeira camada de anticorpos os cortes foram tratados com peróxido de hidrogênio para bloquear a peroxidase endógena e após lavagens repetidas em PBS, foram incubados com soro normal de cabra uma vez que se utîlizou como anticorpo de ligação IgG de cabra anti-IgG de coelho (Miles Yeda Ltd., Israel). Após o tratamento com a terceira camada (Peroxidase anti-Peroxi. 
BARBOSA, A. J. A.; GOBBI, H.: LINO, B. T; LAGES.SILVA, E.; RAMIREZ, L. E.; TEIXEIRA, V. P. A. \& ALMEIDA, H. O. - Estudo comparativo entre o método convencional e o método da peroxidase anti-peroxidase na pesquisa do parasitismo tissular na cardiopatia chagásica crónica. Rev. Inst. Med. trop. São Paulo, 28:91.96, 1986.

dase de coelho, Miles-Yeda Ltd., Israel), os cortes foram tratados com solução de $30 \mathrm{mg} \%$ de tetrahidrocloreto de 3,3-diaminobenzidina com $0,03 \%$ de peróxido de hidrogênio. Como controles positivos utilizaram-se tecidos de camundongo infectado com tripomastigotas, cepa Y, e sacrificados na fase aguda da tripanosomose. Como controles negativos utilizaram-se ao invés do anti-soro primário, soro normal de coelho ou apenas PBS.

Tanto os cortes corados pelo $\mathrm{HE}$ como os corados pela peroxidase anti-peroxidase, foram examinados exaustivamente pelo menos por dois examinadores, sendo todos os ninhos ou figuras suspeitas observadas marcadas na lámina. Os ninhos ou figuras suspeitas que persistiam em dúvida não foram considerados. Deste modo, foi contado o número total de ninhos de amastigotas, diferenciando-se em cortes sequienciais ninhos iguais e diferentes.

\section{RESULTADOS}

Nos très casos examinados, A-804, N-2891 e N-3404, o método da imunoperoxidase para a identificação do T. cruzi apresentou bons resultados (Figs. 1 e 2). Em todos eles o número de ninhos de amastigotas foi sempre maior nas preparaçóes coradas pela imunoperoxidase do que naquelas coradas pelo HE (Tabela I). Mesmo no caso A-804, cujo tecido foi incluído em parafina há 33 anos, os ninhos de amastigotas, que neste caso em particular eram relativamente frequientes, foram corados nitidamente pelo método imunocitoquímico e diagnosticados em quantidade significativamente maior do que nos cortes corados pelo $\mathrm{HE}$. Nas preparações coradas pela peroxidase anti-peroxidase a maioria dos ninhos podia ser facilmente identificada no pequeno ou médio aumento do microscópio; mesmo aqueles de pequenas proporçôes onde somente era possivel observar um ou alguns

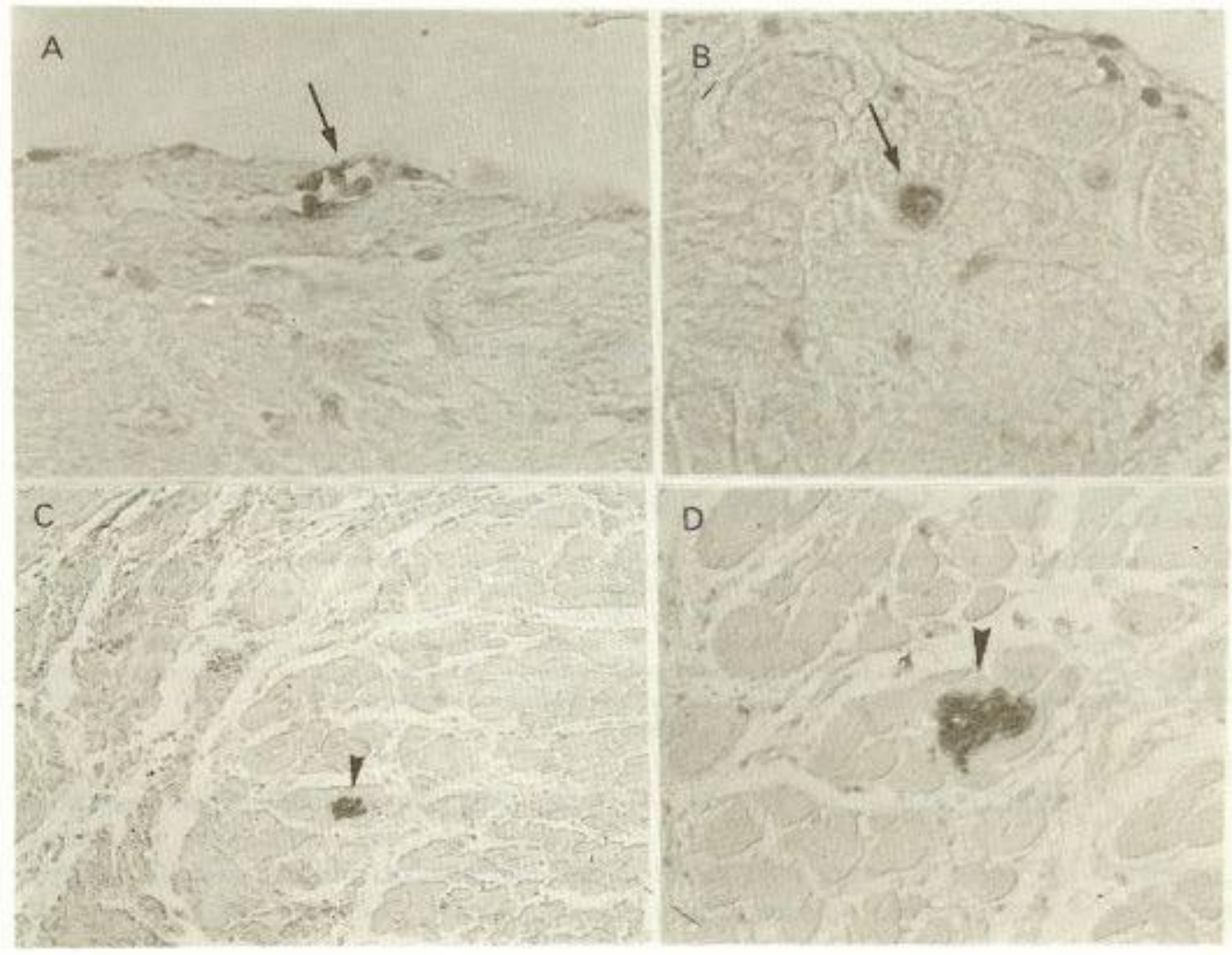

Fig. 1 - Preparaçōes histológicas de miocárdio coradas pelo método đa peroxidase anti-peroxidase para evi. denciar amastigotas do Trypanosoma cruzi em eardiopatia chagásica crỏnica. Contracoloraçāo: Hematoxi lina. Pequenos ninhos de amastigotas fortemente corados (setas), sendo que em A e B apresentam ape nas 4 cu 5 parasitas. Em D observa-se ampliagăo ce C. $A, B$ e $D=500 x ; C=200 x$. 
BARBOSA, A. J. A.; GOBBI, H.; LINO, B. T.; LAGES-SILVA, E.; RAMIREZ, L. E.; TEIXEIRA, V. P. A. \& ALMEIDA, H. O. - Estudo comparativo entre o método convencional e o metodo da peroxidase anti-peroxidase na pesquisa do parasitismo tissular na cardiopatia chagásica cronica. Rev. Inst. Med. trop. Săo Paulo, 28:91-96, 1986.

poucos amastigotas presentes em fibras musculares cortadas obliquamente ou transversalmente e sem nenhuma reação inflamatória circunjacente (Fig, $1 \mathrm{~A}, \mathrm{~B}$ ). Por algumas vezes, (caso A-804 e caso $\mathrm{N}$-3404) identificaram se pelo método da imunoperoxidase, amastigotas de permeio a exsudato inflamatório aparentemente na vizinhança de fibras musculares parcialmente rotas ou no interior de macrófagos (Fig. 2 B, C). Como pode ser deduzido da Tabela I, o resul- tado das contagens expresso em número de ninhos de amastigotas $/ 100$ cortes, corados pelo $\mathrm{HE}$ e pela peroxidase anti-peroxidase (PAP), respectivamente HE/PAP, é: Caso A-804, 80/171; Caso N-3404, 5/116; Caso N-2891, 1/2. Entretanto, muitos desses ninhos se repetiam em cortes sequienciais; o resultado expresso em número de ninhos diferentes por 100 cortes é: Caso A-804, 25/70; Caso N-3404, 3/26 e Caso N-2891, $1 / 2$.

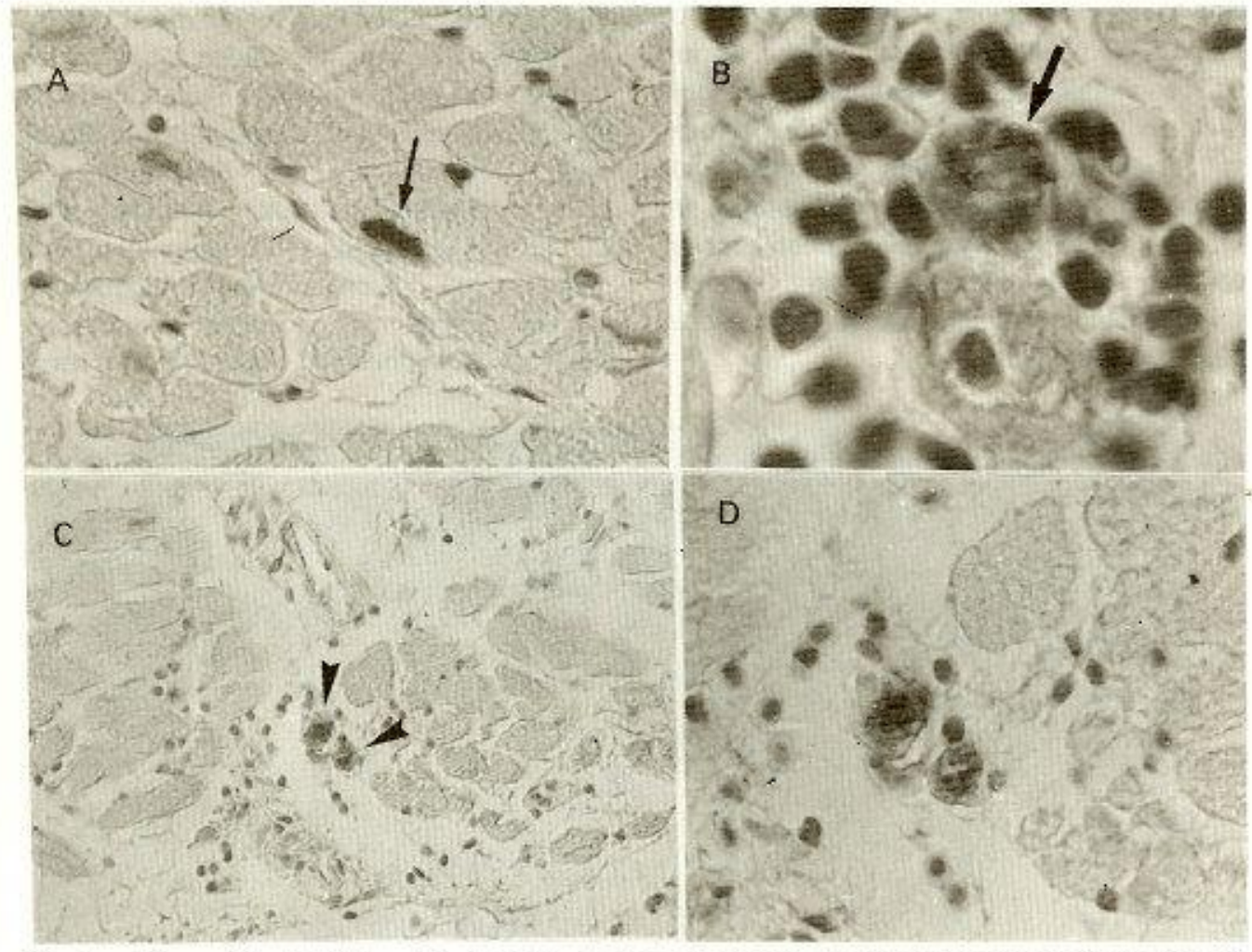

Fig. 2 - Preparaçōes histológicas de miocírdio coradas pelo método da peroxidase anti-peroxidase para exldenciar amastigotas do Trypanosoma cruzi $\mathrm{em}$ cardiopatia chagásica crönica. Contracoloraç̄̄o: Hematoxilina. A) e B) - Pequenos ninhos de amastigotas (setas), em A ocupando apenas parte do sarcoplasma cortado transwersalmente e em B), provavelmente no interior de macrofago, na intimidade de foco inflamatófago. C) Dois pequenos ninhos de amastigotas (setas) $\theta$, como os anteriores, dificilmente identificaveis em preparaçőes coradas convencionalmente. Em D) observa-se ampliaçăo de C. A $\mathrm{e}$. $500 \mathrm{x}$ : $B=1000 x ; C=200 x$.

\section{DISCUSSAO}

A utilizaçăo de método imunocitoquímico especifico para a demonstração de amastigotas do T. cruzi no tecido foi primeiramente empregado por Andrade \& col.4 na tripanosomose experimental através da imunofluorescência indireta. Esta, embora possa se constituir em meio útil para a identificação qualitativa do T. cruzi, apresenta uma série de inconveniências inerentes ao próprio método e amplamente conhecidas ${ }^{12}$. Pode ser útil, por exemplo, para a confirmaçăo da natureza antigênica de acha. dos suspeitos à microscopia de rotina. Entre tanto, o encontro de figuras fluorescentes sus. peitas, quando se pesquisa o parasitismo em 
BARBOSA, A. J. A.; GOBBI, H.; LINO, B. T.; LAGES-SILVA, E.; RAMIREZ, L. E.; TEIXEIRA, V. P. A. \& ALMEIDA, H. O. - Estudo comparativo entre o método convencional a o método da peroxidase anti-peroxidase na pesquisa do parasitismo tissular na cardiopatia chagásica crônica. Rev. Inst. Med. trop. São Paulo, 28:91-96, 1986.

T A B E L A I

Número de ninhos de amastigotas (n. $N$ ) encontrados pelo método de coloraçāo pela Hematoxilina e Eosina (HE) e pelo método da peroxidase anti-peroxidase (PAP) em um mesmo fragmento de tecido de 3 casos de cardiopatia chagásica crônica. n.० $\mathrm{C}=$ número de cortes histológicos.

\begin{tabular}{|c|c|c|c|c|c|c|}
\hline \multirow[t]{2}{*}{ Casos } & \multicolumn{2}{|c|}{ N. ${ }^{\circ}$ de cortes } & \multicolumn{2}{|c|}{ N.o de ninhos } & \multicolumn{2}{|c|}{ Relaçâo n. $.^{\circ} \mathrm{N} / \mathrm{n} .^{\circ}$} \\
\hline & $\mathbf{H E}$ & PAP & $\mathbf{H E}$ & PAP & $\mathbf{H E}$ & PAP \\
\hline A. 804 & 110 & 71 & 87 & 123 & 0,79 & 1,73 \\
\hline$N-3404$ & 100 & 61 & 05 & 71 & 0,05 & 1,16 \\
\hline$N \cdot 2891$ & 100 & 100 & 01 & 02 & 0,01 & 0,02 \\
\hline
\end{tabular}

determinado tecido, torna necessário procederse a coloraçôes rotineiras com finalidade de confirmação ou de localização dos amastigotas. Em virtude desta série de inconveniências, os diversos Autores têm empregado somente as técnicas convencionais de coloração quando se procedem à pesquisa e quantificação do para. sitismo na doença de Chagas humana e na tripanosomose cruzi experimental 1,2,6,11. O grande inconveniente dos métođos convencionais deriva de sua especificidade limitada que se torna ainda mais relevante quando os parasitas săo escassos ou se distanciam de sua morfologia padrăo. Entretanto, com a possibilidade de utilização do método imunocitoquímico pela peroxidase anti-peroxidase, altamente sensivel e específico para a demonstraçăo do T. cruzi em tecidos fixados rotineiramente ${ }^{5}$, abrem-se novas perspectivas para o estudo do parasitismo tis. sular na doença de Chagas. Além disto, o método apresenta duas vantagens: $1 .^{2}$ contra-coloração do tecido que pode ser estudado do ponto de vista morfológico e $2 .^{3}$ a estabilidade da coloraçāo em cortes que podem ser diafanizados, montados entre lâmina e laminula e guardados para exame posterior. Esta série de vantagens da imunoperoxidase tem contribuído para que este método seja empregado em his. toquimica de forma crescente, tanto para a identificação das substâncias antigênicas as mais variadas em tecidos normais e patológicos, como também, de agentes biológicos causadores de doenças, entre estes, alguns parasitas de dificil identificação em preparados rotineiros, como Toxoplasma 8 e Leishmania 10 .

Em relação à pesquisa do $\mathbf{T}$. cruzi, os resultados do presente trabalho demonstram que o método imunocitoquímico empregado parece significativamente mais eficaz que o convencio- nal, mesmo em tecidos conservados em parafina há longo tempo. No material estudado, o método da imunoperoxidase demonstrou amastigotas isolados, ou ninhos de amastigotas que não puderam ser visualizados ou identificados pelo método rotineiro. A análise do material mostrou que estes parasitas não identificados pela coloração convencional foram principalmente aqueles isolados ou formando pequenos aglomerados intracelulares e que apareciam nos cortes constituídos aparentemente por apenas 2, 3 ou 4 parasitas (Fig. 1A, B e Fig. 2). Este fato explica porque nos casos A-804 e N-3404 o número de ninhos de parasitas foi sensivelmente maior nos cortes corados pelo método da imunoperoxidase. Além disto, os dois casos típicos de miocardite chagásica crônica aqui estudados (N-3404 e N-2891), comportaram-se di. ferentemente em relação ao parasitismo. Um deles (N-3404) apresentou rico parasitismo evidenciado pela imunoperoxidase (em média, mais de 1 ninho por corte), enquanto que nas lâminas coradas pelo $\mathrm{HE}$ apresentou, em média, apenas 1 ninho em cada 20 cortes. No outro caso (N-2891), o parasitismo foi raro tanto nas lâminas coradas pelo $\mathrm{HE} \mathrm{(1} \mathrm{ninho} \mathrm{em} 100$ cortes), como naquelas coradas pelo método imunocitoquímico ( 2 ninhos em 100 cortes). Entretanto não podemos afastar que pelo menos parte desta variação do número de parasitas identificados esteja na dependência de variação real da intensidade do parasittsmo nos cortes de tecido e não apenas por serem melhor demonstrados pelo método. Apesar de não ter sido o objetivo deste trabalho avaliar o parasitismo na cardiopatia chagásica crônica, o que exigiria estudo mais abrangente, os resultados aqui encontrados podem constituir.se em mais uma evidência de que em determinado número de casos de cardiopatia chagásica crônica a presença do parasita pode ser muito mais frequien. te e importante do que o correntemente admitido.

Concluindo, os resultados do presente trabalho mostram que, para a identificação e quan. tificação mais precisa do T. cruzi em tecidos de individuos chagásicos, o método imunocitoquímico da peroxidase anti-peroxidase oferece importantes vantagens em relação aos métodos puramente morfologicos, usados habitualmente. 
RARBOSA, A. J. A.; GOBBI, H.; LINO, B. T.; LAGES-SIIVA. E.; RAMIREZ, L. E.; TEIXEIRA, V. R. A. \& ALMEIDA, H. O. - Estudo comparativo entre o método convenclonal e o método da peroxidase anti-peroxddase na pesquisa do parasitismo tissular na cardiopatia chagésica crônica. Rev. Inst. Med. trop. S5á Paulo, 28:91-96, 1986.

\section{SUMMARY}

A comparative study between haematoxilin and Eosin and the peroxidase anti-peroxidase stains for tissular parasitism quantification in chronic chagasic cardiomyopathy.

In most patients with chronic Chagas disease the Trypanosoma cruzl has been shown to be present in extremely scanty numbers in all tissues extensively studied using conventional non-specific histologic techniques presently available. The present work was carried out in order to compare the immunocytochemical method of peroxidase anti-peroxidase (PAP) with the Haematoxilin and Eosin (HE) technique in the quantification of the parasitism in formalin fixed tissues from chronic chagasic patients. Using the same tissue fragment from each one of 3 cases of chronic chagasic car. diomyopathy, sections were obtained for $\mathrm{HE}$ (average: 100 sections per fragment) and for PAP (average: 70 sections per fragment). The fragment from case No. 1 was first studied in 1952 and presented with relatively great number of parasites. The results, expressed as amastigotes nests per 100 sections stained by $\mathrm{HE}$ and PAP 'were, respectively (HE - PAP): case No. 1, $80-171$; case No. 2, 5-116 and case No. 3, 1-2. The present results show that the immunocytochemical method improves the quantitative evaluation of the parasitism in tissues of chronic Chagas disease. Furthemore. it should be preferred not only for the specific diagnosis but also for quantitative studies on the tissular parasitism even in those tissues included in paraffin wax for long periods of time.

\section{REFERENCIAS BIBLIOGRAFICAS}

1. ALMEIDA, H. O.; TEIXEIRA, V. P. A.; GOBBI, H.; ROCHA, A. \& BRANDAO, M. C. - Inflamação asso. ciada a células musculares cardíacas parasitadas pelo Trypanosoma cruzi, em chagásicos crónicos. Arch. bras. Cardiol., 42-43: 183-186, 1984.
2. ALMEIDA, H. C.; TEIXEIRA, V. P. A. \& OLTVEIRA, A. C. F. - Flebite com parasittsmo em supra-renais de chagásicos crônicos. Arch. bras. Cardiop., 36: 341-344, 1981.

3. ANDRADE, Z. A. - Anatomia patológica da doença de Chagas. Rev. golana Med., 4: 103-119, 1958.

4. ANDRADE, Z. A. \& ANDRADE, S. G. - Estudo Imunocitoqufmico da doença de Chagas experimental. Rev. Inst. Med. trop. S. Paulo, 11: 44-47, 1969.

5. BARBOSA, A. J. A. - Método imunocitoqufímico para a identificaçáo de amastigotas do Trypanosoma cruxd em cortes histológicos do roting. Rev. Inst. Med. trop. S. Paulo, 27: 293-297, 1985.

6. BARBOSA Jr., A. A. \& ANDRADE, z. A. - Identifica ção do Trypanosoma cruzd nos tecidos extra-cardíacos de portadores de miocardite crônica chagásíca. Rev. Soc. bras. Med. trop., 17; 123-126, 1984.

7. BOGLiOLO, L. - Patologla. 3.* ed. Rio de Janetro, Guanabars Koogan, 1981. cap. 13, p. 342-395.

8. DUTTON, G. N.; HAY, J. \& RALSTON, J. - The immunocytochemical demonstration of Toxoplasma within the eyes of congenitally infected mice. Amu. trop. Med. Paragit. 78: 431-433, 1984.

9. KRETTLI, A. U.; WEISS-CARRIGTON, P. \& NUSSENZ WEIG, R. S. - Membrane-bound antibodies of bloodstream Trypanosoma cruzl in mice: strain differences in susceptibility to complement-mediated Iysis. Clin. exp. Immunol, 37: 416-423, 1979.

10. LIUMI, N.; ABRAMOWITZ, A.; LONDNER, M.; OKON. E. \& MORAG, A. - Immunoperoxidase method of iden tification of Lelshmania in routinely prepared histo. logical sections. Virchows Arch. path. Anat., 401: 147 . $151,1983$.

11. SOUZA, M. A. \& ALENCAR, A. A. - On the tissular parasitism of Trypanosoms cruxi. $Y$ strain in swiss mice. Rev. Inst. Med, trop. S. Paulo, 26: 316-321, 1984

12. STERNBERGER, L. A. - Immunocytochemistry. and ed. New York, John Wiley and Sons, 1979. Chapter 5 p. 104-169.

Recebrdo pars publicaçăo em 28/6/19as. 